\title{
Lumbar Hernia
}

\author{
Lt Col P Sharma*
}

MJAFI 2009; 65 : 178-179

Key Words : Lumbar hernia; Extra peritoneal mesh repair; Lumbar triangle

\section{Introduction}

L umbar hernias are quite uncommon as compared to other ventral abdominal wall hernias, accounting for less than $1.5 \%$ of all abdominal hernias, with fewer than 300 cases reported over the past 300 years [1]. About $25 \%$ of all lumbar hernias have a traumatic etiology [2]. This may be post-surgical or following blunt injuries associated with intra-abdominal injuries. The management of such patients constitutes a surgical challenge. Clinical diagnosis of this entity is difficult due to non-specific symptoms. The diagnosis is particularly elusive in obese individuals or in post-surgical patients. Though rare defects, lumbar hernias are prone to incarceration and strangulation [1].

\section{Case Report}

A 65 year old male patient presented with gradually increasing painless and reducible swelling in left flank for the past 15 years. There was no history of trauma, previous surgery, hematuria, or altered bowel habits. Over the past six months, the swelling had become irreducible and painful. On admission, there was a $15 \times 15 \mathrm{~cm}$ non-tender, non-pulsatile and irreducible swelling in the left lumbar region with an expansile cough impulse (Fig. 1). The examination of rest of the abdomen, right flank, back and hernial orifices was normal.
Plain radiograph of the abdomen did not reveal any gas shadows within the swelling. Ultrasonography revealed a $12 \times 10 \mathrm{~cm}$ ill defined mass in the left lumbar region with areas of mixed echogenicity within the swelling. Computed tomography (CT) scan of the abdomen was carried out which confirmed the presence of left lumbar hernia containing fatty tissue (Fig. 2). A diagnosis of an irreducible left lumbar hernia was made and patient was taken up for surgery.

The patient was placed in a right lateral position. The swelling was explored through a left flank incision. The retroperitoneal fatty globular mass attached with a tapering pedicle emerging through a narrow constricting ring was discovered to be herniating through the superior lumbar triangle (Fig. 3). The constricting ring was released and the pedicle along with the entire herniated mass was excised.

A prolene mesh was fashioned as inlay prosthesis and was placed in the extra peritoneal space through the defect in the muscle layer. The defect was repaired with prolene continuous sutures and rest of the wound was closed in layers. The immediate postoperative period was uneventful. Oral feeds were started on the second postoperative day. The patient recovered satisfactorily and was discharged after removal of the skin sutures on the eighth postoperative day with a well healed scar. During the follow-up visit after one month, the operation scar was found to be well healed and the patient was absolutely asymptomatic.

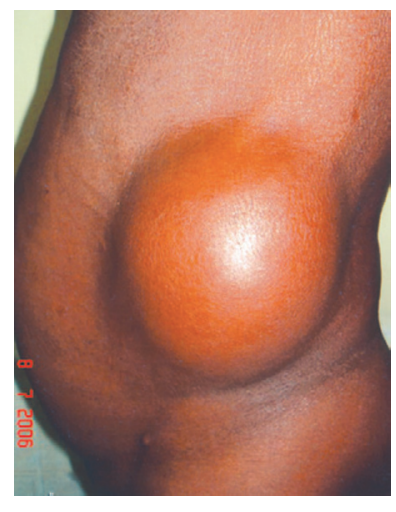

Fig. 1 : Pre operative left lateral view showing lumbar hernia

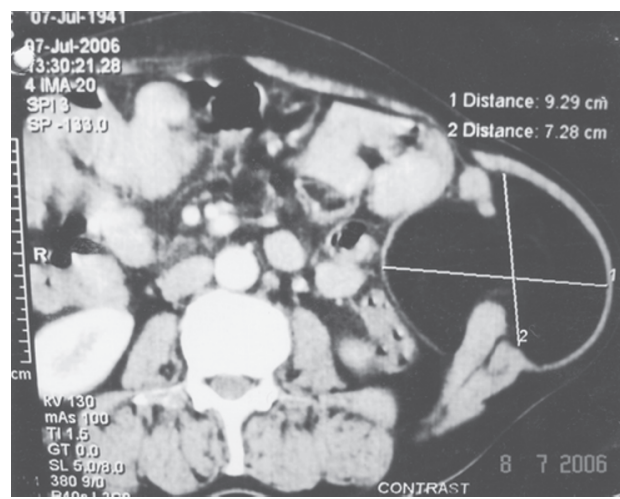

Fig. 2 : CT scan of the abdomen shows a left lumbar hernia

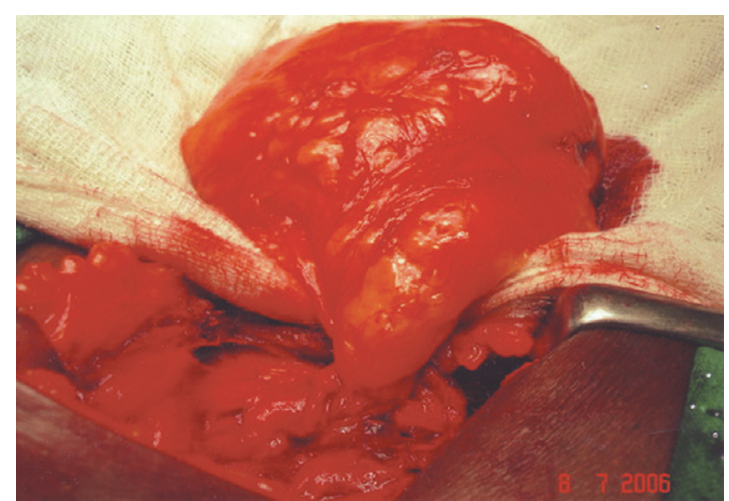
Fig. 3 : Intra operative view showing herniated fatty mass "Graded Specialist (Surgery), Indian Level-III Hosp, UN Mission Congo (MONUC), C/o-301 Inf Bde Gp, C/o-56 APO. Received : 20.10.06; Accepted : 17.07 .08 E-mail : pawansharma 5@rediffmail.com 


\section{Discussion}

Lumbar hernias are relatively rare. These occur more commonly in males and are twice as common on the left than the right side. Patients are usually between 50 to 70 years old. These hernias can occur anywhere within the lumbar region but are more common through the superior lumbar triangle (of Grynfeltt-Lesshaft), an inverted triangle bounded by $12^{\text {th }}$ rib, erector spinae and the posterior border of the internal oblique muscle. The inferior lumbar triangle (of Petit), bounded by iliac crest, posterior border of external oblique and the anterior border of latissimus dorsi is the next most frequent site [2]. Lumbar hernias have been classified as congenital $(20 \%)$ or acquired $(80 \%)$. If acquired, they may be primary $(55 \%)$ or secondary following trauma, surgery or inflammation $(25 \%)$.

Patients are usually asymptomatic but may complain of backache, flank pain or a dragging sensation. These hernias have a natural history of a gradual increase in size over time and may assume large proportions [3]. The differential considerations at this stage include lipoma, soft tissue tumors, hematoma or abscess. The hernia may contain retroperitoneal fat, kidney, colon or less commonly small bowel, omentum, ovary, spleen or appendix. On auscultation, bowel sounds may be audible over the swelling if the hernia contains bowel loops. In obese patients detection of a mass is particularly difficult. Bowel incarceration occurs in $25 \%$ but strangulation is rare because of wide hernial neck [4].

Lateral or oblique radiograph of the lumbar region may show gas filled loops of the bowel lying outside the abdominal cavity. Upper or lower gastrointestinal contrast studies are useful in delineating the herniated bowel segment. In addition, an intravenous urogram may be performed to visualize any displacement of the kidney or ureter into the hernia. Ultrasonography may fail to demonstrate the hernia due to low index of suspicion and presence of fat. CT scan can accurately distinguish the muscular and fascial layers, detect the presence of a defect in these layers, visualize herniated fat (Fig. 2) or viscera and differentiate a hernia from a hematoma, abscess or soft-tissue tumor $[5,6]$.

The goal of hernia repair is to eliminate the defect and to construct an elastic and firm abdominal wall that will withstand the stress of daily physical activities. A lumbar hernia should be repaired surgically, as it is prone to both obstruction and strangulation [7,8]. A wide variety of techniques have been described for repair of lumbar hernias. These include anatomical closure, overlapping of the aponeuroses, use of musculofascial flaps, prosthetic meshes and laparoscopic mesh repair in case of uncomplicated lumbar hernias [4].

Currently, extraperitoneal mesh repair is considered the optimal treatment for isolated unilateral lumbar hernia. The Rives Stoppa approach, wherein, a large rectangular mesh is fashioned to be placed in the pre peritoneal space extending from umbilicus to retropubic space and between the two anterior superior iliac spines, appear to be the most promising open technique for bilateral lumbar hernia, recurrent hernias or multiple site hernias with comparatively low recurrence rates [8]. Extra- peritoneal position of the mesh is advantageous as no bony anchorage is essential. The weight of the intraperitoneal contents is an additional support to maintain the mesh in correct position in the early postoperative period. Laparoscopic transabdominal preperitoneal mesh repair for lumbar hernia confers all the benefits of minimal access surgery to the patient. It is a tensionless repair. It follows the current principle of hernia surgery and is based on the sound physiological principle of diffusing the total intra-abdominal pressure on each square inch of the mesh implanted $[9,10]$.

\section{Conflicts of Interest}

None identified

\section{References}

1. Russell RCG, Norman S, editors. Bailey \& Love's Short Practice of Surgery. $24^{\text {th }}$ edn. London :Edward Arnold $2004 ; 1287$.

2. Skandilakis JE, Flament JB. The Surgical Clinics of North America 2000; 80: 388-91.

3. Geis WP, Hodakowski GT. Lumbar hernia. In: Nyhus L, Condon R, editors. Hernia. $5^{\text {th }}$ ed. Philadelphia 2001; 425-7.

4. Devlin B, Kingsnorth AN. Management of abdominal hernias. $2^{\text {nd }}$ edn. Edward Arnold, London $1998 ; 330-4$.

5. Baker ME, Weinerth JL, Andriani RT, et al. Lumbar Hernia: diagnosis by CT. Am J Roentgenol 1987; 148: 565-7.

6. Killeen KL, Girard S, Demeo JH, et al. Use of CT to diagnose traumatic lumbar hernia. Am J Roentgenol 2000; 174:1413-5.

7. Leber GE, Garb JL, Albert Al, Reed WP. Long-term complications associated with prosthetic repair of incisional hernias. Arch Surg 1998; 132:1141-4.

8. Meinke AK. Totally extraperitoneal laparoendoscopic repair of lumbar hernia. Surg Endosc 2003; 17: 734-7.

9. Heniford BT, Iannitti DA, Gagner M. Laparoscopic inferior and superior lumbar hernia repair. Arch Surg 1997;132:1141-4.

10. Chowbey PK, Sharma A, Khullar R, et al. Laparoscopic Ventral hernia repair. J Laparoendosc Adv Surg Tech 2000; 10:79-84. 